\title{
Comparison of two methods for magnetic field synthesis on a solenoid's axis
}

\author{
Marcin Ziolkowski
}

Received: 16 March 2010 / Accepted: 10 May 2011 / Published online: 26 May 2011

(C) The Author(s) 2011. This article is published with open access at Springerlink.com

\begin{abstract}
Magnetic field synthesis problems have been discussed at length in scientific literature but they still remain as a topic of research in electrical engineering, physics and medical applications. In these disciplines, there is often a need to design an electromagnetic arrangement which can generate a magnetic field of required distribution. Such an arrangement can also work as an active shield. The aim of an active shield is to generate a specified magnetic field which counteracts the external magnetic fields in a protected region. The idea of active shielding is to construct a suitable arrangement of coils, excited with currents that generate an opposite magnetic field sufficient to cancel out the unwanted external fields. The opposite field must have the same frequency and amplitude as the external field. If the incident field presents a wide bandwidth, the final aim is to generate an opposite field in the same frequency range or at least in a range as large as possible. Two independent methods of magnetic fields synthesis, i.e. iteratively regularized Gauss-Newton method and Genetic Algorithm coupled with Bezier curves-based method, are discussed and compared in this paper.
\end{abstract}

Keywords Magnetic field synthesis - Nonlinear inverse problems · Iterative methods - Genetic algorithms .

Active shields

\section{Introduction}

Magnetic field synthesis problems have been discussed at length in scientific literature but they still remain as a topic

M. Ziolkowski ( $\square)$

Faculty of Electrical Engineering,

West Pomeranian University of Technology,

Sikorskiego 37, 70-313 Szczecin, Poland

e-mail: marcin.ziolkowski@zut.edu.pl of research in electrical engineering, physics and medical applications. In these disciplines, there is often a need to design an electromagnetic arrangement which can generate a magnetic field of required distribution. These problems are especially essential in various biomedical applications, e.g. in Magnetic Resonance Imaging [1,2]. Such an arrangement can also serve as an active shield [2]. The aim of an active shield is to generate a specified magnetic field which counteracts the external magnetic fields in a protected region. The idea of active shielding is to construct a suitable arrangement of coils, excited with currents that generate an opposite magnetic field sufficient to cancel out the unwanted external fields. The opposite field must have the same frequency and amplitude as the external field. If the incident field presents a wide bandwidth, the final aim is to generate an opposite field in the same frequency range or at least in a range as large as possible [3]. This paper is a continuation of [4,5] and discusses the problem of finding the shape of a solenoid which produces a given field on the axis. In mathematics such problems belong to the linear or nonlinear ill-posed inverse problems. To solve them, special numerical algorithms must be applied. Two independent methods, i.e. iteratively regularized Gauss-Newton method and genetic algorithm (GA) coupled with Bezier curves-based method are discussed and compared in this paper.

\section{Problem description}

Let us assume, we need to produce a magnetic field free region in a static uniform magnetic field. The external field is in the direction of the $z$-axis and is given by the equation $\boldsymbol{H}=-H_{0} \mathbf{1}_{z}$. The protected region lies near the solenoid's symmetry axis ( $z$-axis in cylindrical coordinate system). Our task is to find the shape of a solenoid, so that the magnetic 
Fig. 1 On the left-a definition sketch for a solenoid of thickness $t$ and unknown shape $r^{\prime}=f\left(z^{\prime}\right)$; on the right-3D visualization

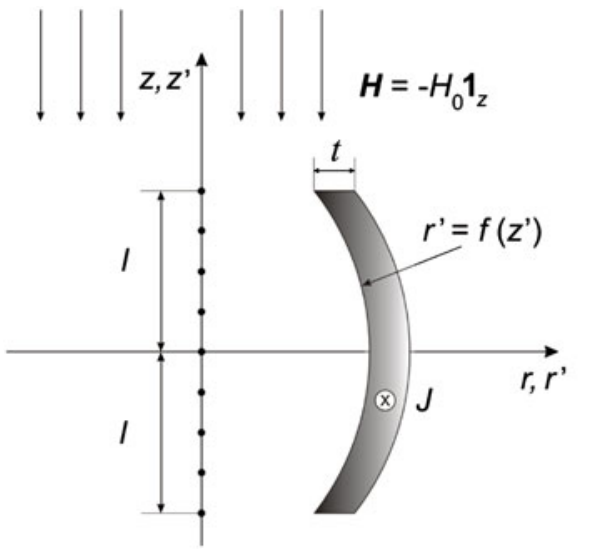

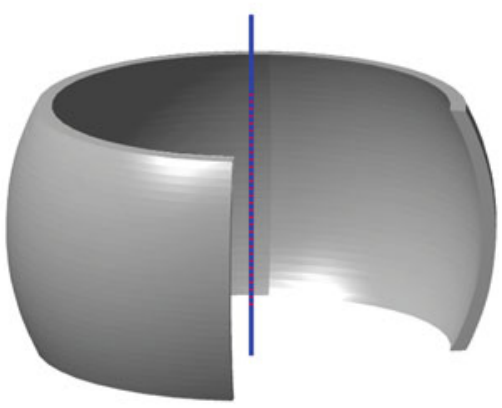

field intensity on the axis is uniform but in opposite direction to the external field $\left(H=H_{0} \mathbf{1}_{z}\right.$ for $\left.-l \leq z \leq l\right)$. The inner and outer surfaces of the solenoid are created by the rotation of curves $r=f(z)$ and $r=f(z)+t$ around the $z$-axis, respectively. The solenoid is of height $2 l$ and contains a large number $N$ of tightly wound turns of wire carrying current $I$, then there is an effective current density $J$ within the solenoid, where $J=N I /(2 l t)$. In such a case, the actual solenoid can be replaced by a region carrying a constant current density $J$ (the assumption becomes more accurate as $N$ is increased). The considered arrangement is shown in Fig. 1.

By using the formula for the magnetic field intensity on the circular loop axis, it can be shown that the axial component of the magnetic field on the solenoid axis has the simple form (only $z$-component exists) [6]:

$H_{z}(z)=\frac{J}{2} \int_{-l}^{l} \int_{f\left(z^{\prime}\right)}^{f\left(z^{\prime}\right)+t} \frac{r^{\prime 2} \mathrm{~d} r^{\prime} \mathrm{d} z^{\prime}}{\left[\sqrt{r^{\prime 2}+\left(z-z^{\prime}\right)^{2}}\right]^{3}}$.

Here, primed variables refer to source points within the solenoid, and the unprimed variable $z$ denotes field points on the $z$-axis. The integral with respect to $r^{\prime}$ may be evaluated in closed form and the result is:

$$
\begin{aligned}
& H_{z}(z)=\frac{J}{2} \int_{-l}^{l}\left\{\ln \frac{f\left(z^{\prime}\right)+t+\sqrt{\left[f\left(z^{\prime}\right)+t\right]^{2}+\left(z-z^{\prime}\right)^{2}}}{f\left(z^{\prime}\right)+\sqrt{f\left(z^{\prime}\right)^{2}+\left(z-z^{\prime}\right)^{2}}}\right. \\
& +\frac{f\left(z^{\prime}\right)}{\sqrt{f\left(z^{\prime}\right)^{2}+\left(z-z^{\prime}\right)^{2}}} \\
& \left.-\frac{f\left(z^{\prime}\right)+t}{\sqrt{\left[f\left(z^{\prime}\right)^{2}+t\right]+\left(z-z^{\prime}\right)^{2}}}\right\} \mathrm{d} z^{\prime} .
\end{aligned}
$$

Finally, for desired magnetic field $H_{0}$ equal to $H_{z}$ yields:

$\frac{2 H_{0}}{J}=\int_{-l}^{l} K\left[z, z^{\prime}, f\left(z^{\prime}\right)\right] \mathrm{d} z^{\prime}$.
The Eq. (3) is a nonlinear Fredholm equation of the first kind with the unknown function $f\left(z^{\prime}\right)$, where the form of $K$ is evident from (2). Its solution gives the required shape of the solenoid. The Fredholm equation of the first kind belongs to the class of ill-posed inverse problems as defined by Hadamard. These problems have been examined in numerous papers, mainly by Tikhonov et al. [7]. The similar magnetic field synthesis problems have been considered in the past as well [5,6,8-10], however, the considered arrangements and numerical procedures of calculation of the optimal solenoids have been defined in a different way. In [5], a magnetic field synthesis on the axis of a different cylindrical solenoid's shape has been considered. In papers $[6,8,9]$, authors assumed that a magnetic field with the desired distribution is generated by a cylindrical sectional solenoid with different current densities in each section, and a consideration of a solenoid with infinitely thin winding was the subject of [10]. The problem studied in this paper is nonlinear and requires a different approach. Two different methods have been proposed and utilized to find the optimum shape of the solenoid, namely: iteratively regularized Gauss-Newton method and GA coupled with Bezier curves-based method.

\subsection{Iteratively regularized Gauss-Newton method}

Since the problem is nonlinear, the construction and especially the analysis of regularization is not a simple task and in such a case there is no straightforward way to obtain a successful solution. For some cases, the well-known Tikhonov regularization methods could be applied, however, finding a global minimizer to a nonlinear inverse problem is not trivial. The functional to be minimized in this method may have many local minima and there is still a risk that an obtained solution is not correct or not stable. In these cases, the iterative methods are an attractive alternative [11]. In this paper, the iteratively regularized Gauss-Newton method has been used. This method gives additional stabilization for the solution of (3). Rewriting the nonlinear Eq. (3) into a matrix form yields: 
$\boldsymbol{K}(\boldsymbol{f})=\boldsymbol{h}$,

where $\boldsymbol{h}=2 \boldsymbol{H}_{0} / \boldsymbol{J}$, and $\boldsymbol{f}$-unknown vector to be found.

The solution of (4) is equivalent to the minimizing of the following functional:

$\|\boldsymbol{K}(\boldsymbol{f})-\boldsymbol{h}\|=\min$.

Instead of minimizing the functional (5), the iteratively regularized Gauss-Newton method can be applied, as follows [11]:

$$
\begin{aligned}
\boldsymbol{f}^{k+1}=\boldsymbol{f}^{k} & +\left\{\boldsymbol{K}^{\prime}\left(\boldsymbol{f}^{k}\right) \cdot \boldsymbol{K}^{\prime}\left(\boldsymbol{f}^{k}\right)+\alpha^{k} \boldsymbol{L}\right\}^{-1} \\
& \times\left\{\boldsymbol{K}^{\prime}\left(\boldsymbol{f}^{k}\right) \cdot\left[\boldsymbol{h}-\boldsymbol{K}\left(\boldsymbol{f}^{k}\right)\right]\right\}-\alpha^{k}\left(\boldsymbol{f}^{k}-\boldsymbol{f}^{0}\right),
\end{aligned}
$$

where $\boldsymbol{f}^{k}$-values of the unknown vector $\boldsymbol{f}$ in successive iteration, $k$-the number of current iteration, $\alpha^{k}$-regularization parameters as a sequence of numbers, $\boldsymbol{L}$-identity diagonal matrix, $f^{0}$-initial values for the unknown vector $f$, and $\boldsymbol{K}^{\prime}(\boldsymbol{f})=\partial \boldsymbol{K}(\boldsymbol{f}) / \partial \boldsymbol{f}$-matrix of derivatives which can be calculated analytically from (2):

$$
\begin{aligned}
K^{\prime}(f)= & \frac{\partial K(f)}{\partial f} \\
= & \frac{\left[f\left(z^{\prime}\right)+t\right]^{2}}{\left\{\sqrt{\left[f\left(z^{\prime}\right)+t\right]^{2}+\left(z-z^{\prime}\right)^{2}}\right\}^{3}} \\
& -\frac{f\left(z^{\prime}\right)^{2}}{\left[\sqrt{f\left(z^{\prime}\right)^{2}+\left(z-z^{\prime}\right)^{2}}\right]^{3}} .
\end{aligned}
$$

The choice of the sequence of regularization parameters $\alpha^{k}$ is crucial for the stability and accuracy of the iterative procedure (6). In this paper, it is assumed that $\alpha^{k}$ is the monotonically decreased sequence of positive numbers satisfying: $0<\alpha^{k+1} \leq \alpha^{k}$ and $\lim _{k \rightarrow \infty} \alpha^{k}=0$ [12]. Another problem is the criterion allowing determining the optimal value of the regularization parameter $\alpha_{\text {opt }}$. Several approaches to obtain optimal $\alpha_{\text {opt }}$ in linear inverse problem are known. The common approach is for example $L$-curve method (LCM) proposed by Hansen [13] and applied for instance in [14]. Another well-known scheme can be general cross validation method (GCV) introduced in [15]. However, for nonlinear inverse problems rigorous procedures to determine the optimal value of the regularization parameter are rarely available [16]. The $L$-curve as a logarithmic plot of the residual norm $\|\boldsymbol{K}(\boldsymbol{f})-\boldsymbol{h}\|$ versus $\|\boldsymbol{f}\|$ for different values of $\alpha$ could not be obtained for the nonlinear inverse problem considered in this paper. It coincides with the results presented in $[17,18]$. In the current problem the discrepancy principle method for a rigorous choice of the regularization parameter $\alpha$ has been used. This method bases on the plot of the residual norm || $\boldsymbol{K}(\boldsymbol{f})-\boldsymbol{h} \|$

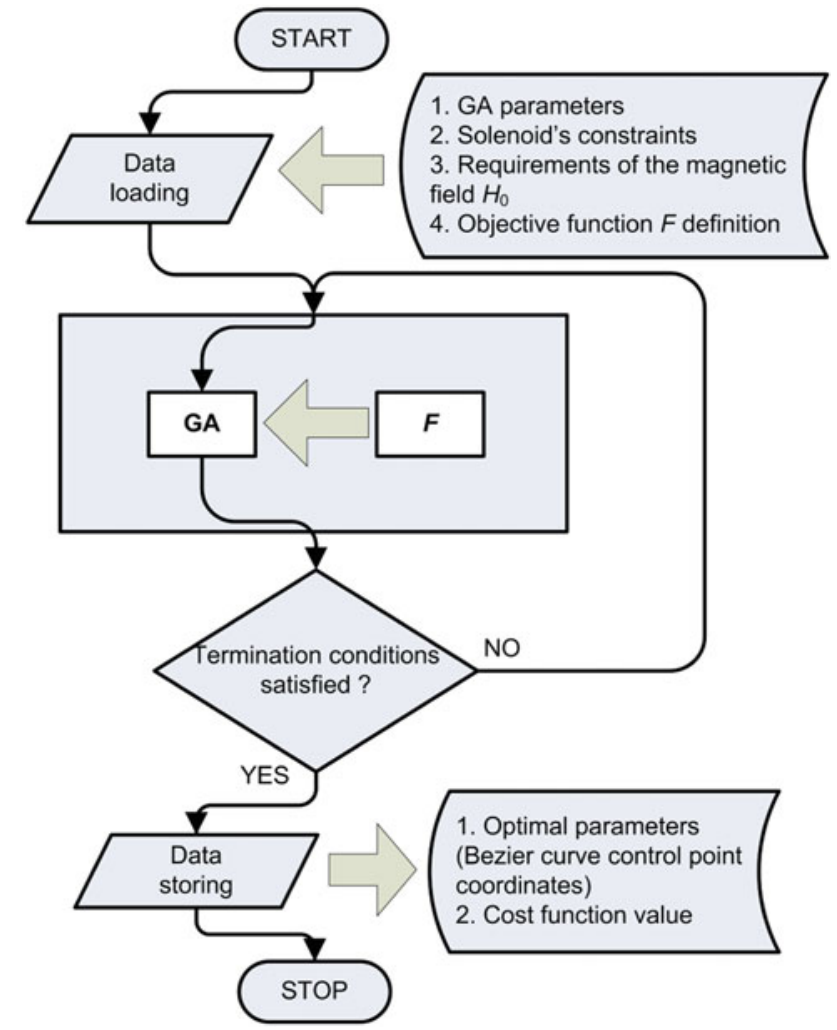

Fig. 2 A flowchart of the optimization procedure based on genetic algorithm (GA) and Bezier curves

Table 1 Search space (minimal and maximal values of all parameters under consideration)

\begin{tabular}{llll}
\hline \multicolumn{2}{l}{ Parameter } & Lowest value $(\mathrm{cm})$ & Highest value $(\mathrm{cm})$ \\
\hline$C_{0}$ & $r_{0}$ & 0 & 100 \\
& $z_{0}$ & 6.25 & 10 \\
$C_{1}$ & $r_{1}$ & 0 & 100 \\
& $z_{1}$ & 3.75 & 7.50 \\
$C_{2}$ & $r_{2}$ & 0 & 100 \\
& $z_{2}$ & 2.5 & 6.25 \\
$C_{3}$ & $r_{3}$ & 0 & 100 \\
& $z_{3}$ & 0 & 5.0 \\
\hline
\end{tabular}

versus regularization parameter $\alpha$. Next, the optimal regularization parameter $\alpha_{\text {opt }}$ is chosen for which the residual curve plot intersects the horizontal line representing the noise level $[11,18]$. In noiseless case (considered in this paper), the optimal value is determined for the minimal value of the residual norm $\|\boldsymbol{K}(\boldsymbol{f})-\boldsymbol{h}\|$.

\subsection{Genetic algorithms and Bezier curve-based method}

Another method which can be applied for finding the function $f(z)$, couples the basic formula (3) with a GA and Bezier 
Fig. 3 On the left-logarithmic plot of the residual norm $\|\boldsymbol{K}(\boldsymbol{f})-\boldsymbol{h}\|$ versus regularization parameter $\alpha$, $x$-position corresponding to the optimal parameter $\alpha_{\text {opt }}=0.0435$; on the right-relative error $\delta(\%)$ on the solenoid's axis calculated for $\alpha_{\text {opt }}$; desired magnetic field $H_{0}=1,500 \mathrm{~A} / \mathrm{m}$
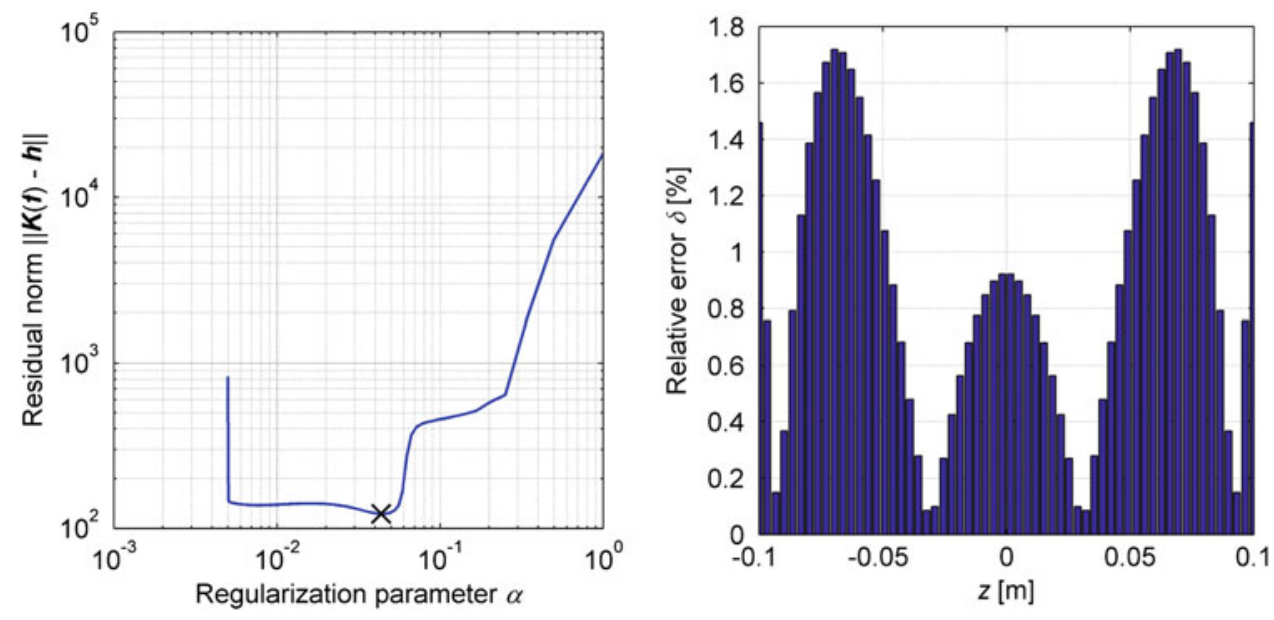

curves. In this case, it is assumed that the inner and outer surfaces of the solenoid are generated by rotating plane Bezier curves $r=f_{B}(z)$ and $r=f_{B}(z)+t$ about the $z$-axis, respectively. A plane Bezier curve can be defined by the following equation $[19,20]$ :

$$
\left\{\begin{array}{l}
r(p) \\
z(p)
\end{array}\right\}=\sum_{i=0}^{w}\left\{\begin{array}{l}
r_{i} \\
z_{i}
\end{array}\right\} B_{i, w}(p),
$$

where $r_{i}, z_{i}$ are coordinates of Bezier curve control points $C_{i}\left(r_{i}, z_{i}\right), B_{i, w}(p)$-Bernstein polynomials and $p \in[0,1]$.

The first few Bernstein polynomials are:

$$
\begin{aligned}
& B_{0,0}(p)=1, \quad B_{0,1}(p)=1-p, \quad B_{1,1}(p)=p, \\
& B_{0,2}(p)=(1-p)^{2}, \\
& B_{1,2}(p)=2(1-p) p, B_{2,2}(p)=p^{2}, \\
& B_{0,3}(p)=(1-p)^{3}, \\
& B_{1,3}(p)=3(1-p)^{2} p, \quad B_{2,3}(p)=3(1-p) p^{2}, \\
& B_{3,3}(p)=p^{3} .
\end{aligned}
$$

Coordinates $r_{i}$ and $z_{i}$ of the Bezier curve control points $C_{i}$ are the design parameters. The optimization process is a determination of the parameters $r_{i}$ and $z_{i}$, which ensures a minimum of the cost function, which is obtained from the desired magnetic field values (equal to $H_{0}$ ) and the calculated ones at $n$ points along the axis of the solenoid. The cost function is given by (root mean square error):

$F=\sqrt{\sum_{j=1}^{n}\left(H_{0, j}-H_{\text {calculated }, j}\right)^{2}}$.

It is known that in a uniform axi-symmetrical magnetic field only half of the solenoid has to be optimized. In this case only the upper part of the solenoid is considered for optimization purposes, while the lower part of the solenoid is built taking into account the symmetry plane of the solenoid. The flowchart of the optimization procedure is shown in Fig. 2.

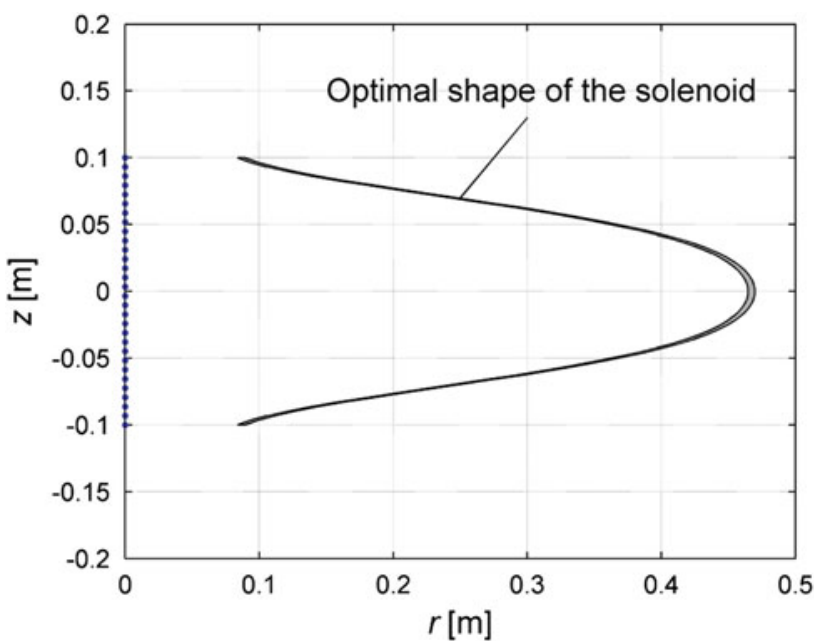

Fig. 4 Optimal shape of the solenoid for desired $H_{0}=1,500 \mathrm{~A} / \mathrm{m}$, $\alpha_{\text {opt }}=0.0435$

As a search tool, a modified version of the GA presented in [21] has been implemented in MATLAB.

At the beginning of the optimization process, GA parameters, requirements of the magnetic field, cost function $F$, and constraints of the solenoid are defined. Next, the initial population is randomly generated. Cost functions are calculated for all the candidates. A selection process then takes place. It is based on roulette-wheel selection. In all cases, the selection coefficient is equal to 0.5 which means that half of the population size goes to the crossover process. After the crossover process, mutation takes place. This relies on random changes in candidates. The aim of the mutation is to improve candidates, making them into better solutions. The mutation coefficient should not be high and in all the cases has been set to 0.2 . Promising candidates go to the next generation and become a new set of candidate solutions. This process repeats until it stops after reaching a maximum number of 
Fig. 5 On the left-logarithmic plot of the residual norm $\|\boldsymbol{K}(\boldsymbol{f})-\boldsymbol{h}\|$ versus regularization parameter $\alpha$, $x$-position corresponding to the optimal parameter $\alpha_{\text {opt }}=0.0833$; on the right-relative error $\delta(\%)$ on the solenoid's axis calculated for $\alpha_{\text {opt }} ;$ desired magnetic field $H_{0}=2,000 \mathrm{~A} / \mathrm{m}$

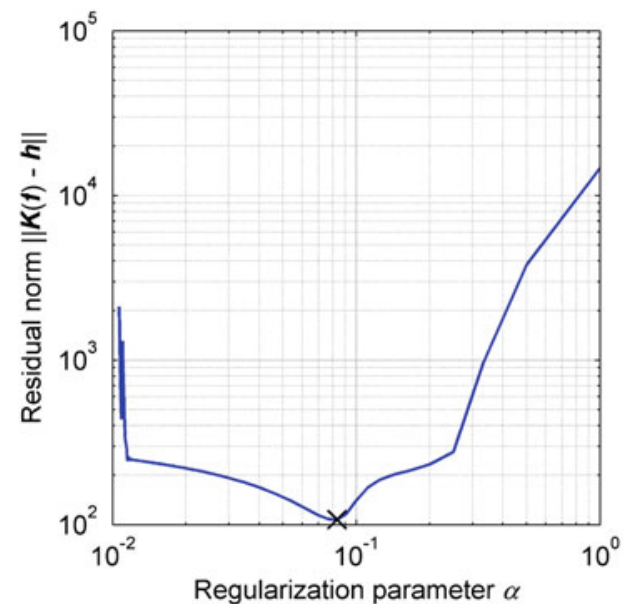

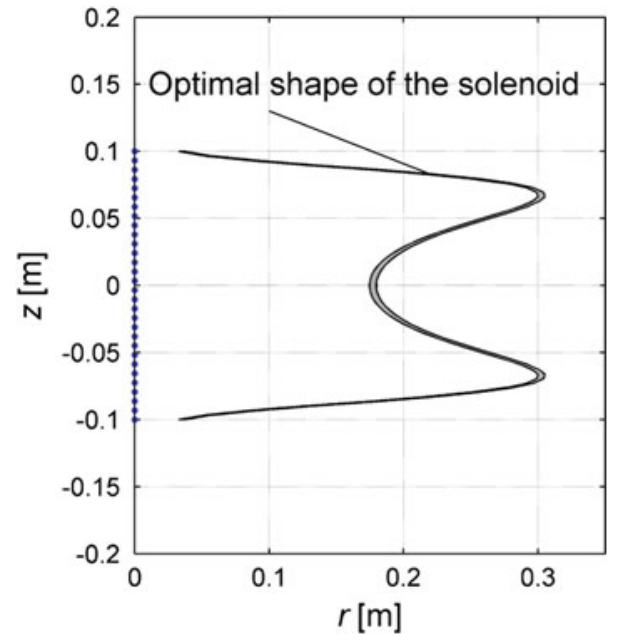

Fig. 6 Optimal shape of the solenoid for desired $H_{0}=2,000 \mathrm{~A} / \mathrm{m}$, $\alpha_{\text {opt }}=0.0833$

iterations. There are eight design parameters in the current optimization problem: $r_{i}, z_{i}$, where $i=0,1,2,3$. All possible values of the parameters under consideration are shown in Table 1. Exactly 32,000 feasible solutions in the optimization problem have been calculated. The total number of generations was equal to 1,000 .

\section{Numerical results}

The number of discretization points $n$ in (4) for magnetic field calculation as well as for $f\left(z^{\prime}\right)$ curve discretization in both methods was set to 60 . The chosen value is the result of compromise between the assumed accuracy and duration of calculations. The calculations have been made for $l=10 \mathrm{~cm}$, $t=5 \mathrm{~mm}$, and $N I=1,000$ Ampere-turns.

\subsection{Results for the iteratively regularized Gauss-Newton method}

Several schemes for determining the sequence of parameters $\alpha^{k}$ are known, however, the simplest choice rule assumes that the $\alpha^{k}$ are the terms of a geometric or harmonic sequence [12]. In this paper, it is assumed that the successive term of
Fig. 7 On the left-logarithmic plot of the residual norm $\|\boldsymbol{K}(\boldsymbol{f})-\boldsymbol{h}\|$ versus regularization parameter $\alpha$, $x$-position corresponding to the optimal parameter $\alpha_{\mathrm{opt}}=0.04$; on the right-relative error $\delta$ (\%) on the solenoid's axis calculated for $\alpha_{\mathrm{opt}}$; desired magnetic field $H_{0}=2,500 \mathrm{~A} / \mathrm{m}$
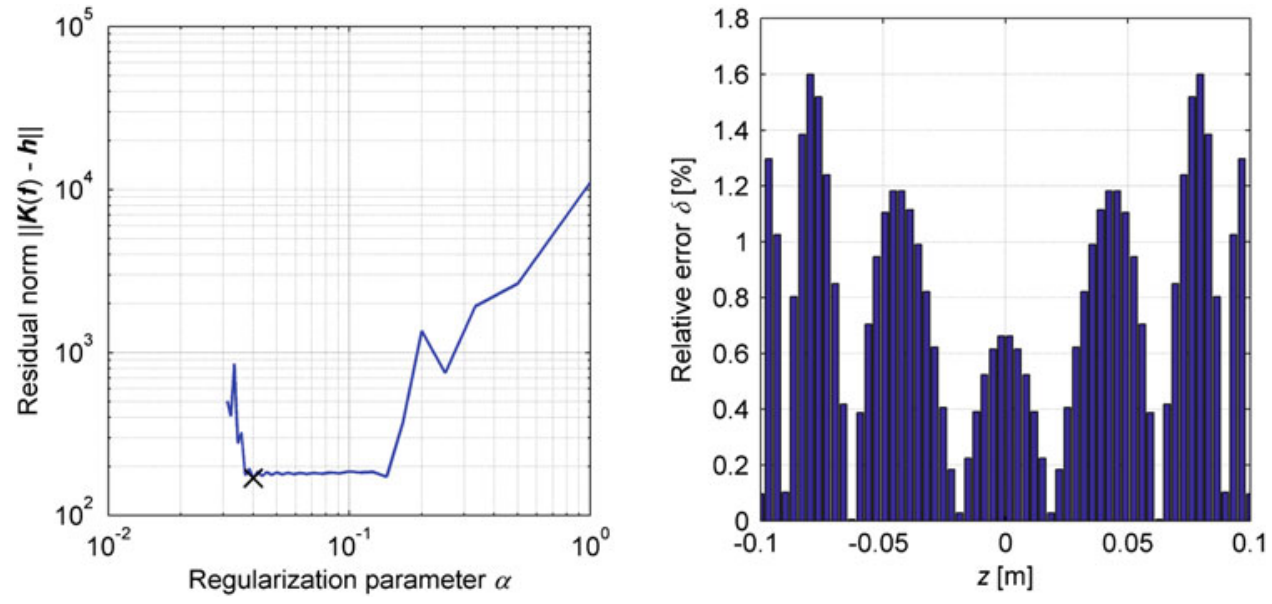


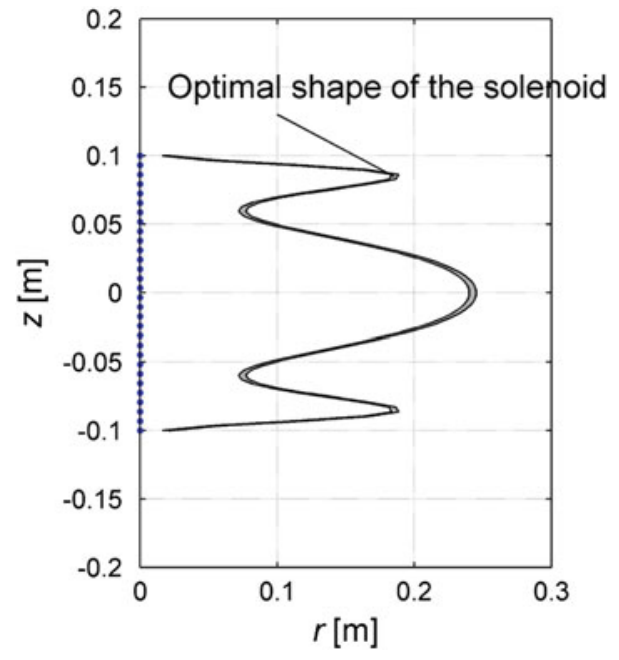

Fig. 8 Optimal shape of the solenoid for desired $H_{0}=2,500 \mathrm{~A} / \mathrm{m}$, $\alpha_{\text {opt }}=0.04$

regularization parameter is equal to $\alpha^{k}=1 / k$, where $k$ is number of iteration in (5). The total number of iterations has been set to 200. The calculations have been performed for three exemplary values of desired magnetic field on the axis, i.e. $H_{0}=1,500,2,000$ and 2,500 A/m, respectively. There are two figures which present complete set of results obtained for each value of desired magnetic field $H_{0}$. For example, in Fig. 3 on the left, logarithmic of the residual norm $\| \boldsymbol{K}(\boldsymbol{f}-\boldsymbol{h} \|$ versus regularization parameter $\alpha$ for $H_{0}=1,500 \mathrm{~A} / \mathrm{m}$ has been presented. The minimal value of $\| \boldsymbol{K}(\boldsymbol{f}-\boldsymbol{h} \|$ corresponding to the optimal value of regularization parameter $\alpha_{\mathrm{opt}}$ is marked by cross. In Fig. 3 on the right, the relative error $\delta$ on solenoid's axis for calculated $\alpha_{\text {opt }}$ is shown. The relative error $\delta$ is calculated using the following formula:

$\delta[\%]=\frac{\left|H_{\text {calculated }}-H_{0}\right|}{H_{0}} \cdot 100$
Table 2 Summarized results obtained using iteratively regularized Gauss-Newton method

\begin{tabular}{llll}
\hline$H_{0}(\mathrm{~A} / \mathrm{m})$ & $\alpha_{\mathrm{opt}}$ & $\delta_{\text {mean }}(\%)$ & $\delta_{\max }(\%)$ \\
\hline 1,500 & 0.0435 & 0.91 & 1.71 \\
2,000 & 0.0833 & 0.57 & 1.52 \\
2,500 & 0.04 & 0.75 & 1.60 \\
\hline
\end{tabular}

Table 3 The relative errors $\delta$ for different values of discretization points $n$ for $H_{0}=1,500 \mathrm{~A} / \mathrm{m}$

\begin{tabular}{llc}
\hline$n$ & $\delta_{\text {mean }}(\%)$ & $\delta_{\max }(\%)$ \\
\hline 5 & 8.41 & 12.61 \\
15 & 1.57 & 2.26 \\
30 & 0.92 & 1.77 \\
60 & 0.91 & 1.71 \\
100 & 0.91 & 1.69 \\
\hline
\end{tabular}

In Fig. 4, the optimal shape of the solenoid for $H_{0}=$ $1,500 \mathrm{~A} / \mathrm{m}$ has been shown.

Figures 5-8 show the results of calculations for all the remaining desired values of $H_{0}$.

It can be seen that optimal values of regularization parameters $\alpha_{\text {opt }}$ are different for different desired magnetic field $H_{0}$, however, the relative error $\delta$ is practically on the same level and its maximal value does not exceed $1.8 \%$. From the Figs. 4, 6 and 8, it can be observed that the obtained shapes of the solenoids are different. The simplest shape is for desired magnetic field $H_{0}=1,500 \mathrm{~A} / \mathrm{m}$, whereas the most complex is for $H_{0}=2,500 \mathrm{~A} / \mathrm{m}$. The obtained results are summarized in Table 2.

In order to check the accuracy, the calculations have been performed also for different values of discretization points $n$. The relative errors $\delta$ for different values of discretization points $n$ obtained for desired $H_{0}=1,500 \mathrm{~A} / \mathrm{m}$ are presented in Table 3.
Fig. 9 On the left-cost function $F$ values after succeeding generations of GA for desired $H_{0}=1,500 \mathrm{~A} / \mathrm{m}$; on the right-relative error $\delta(\%)$
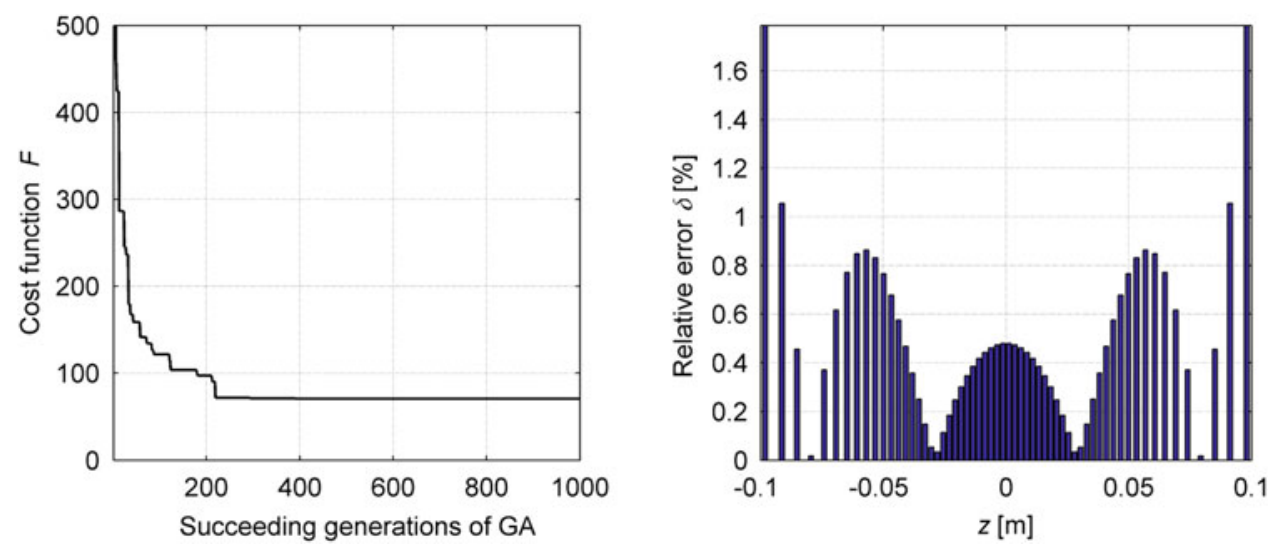


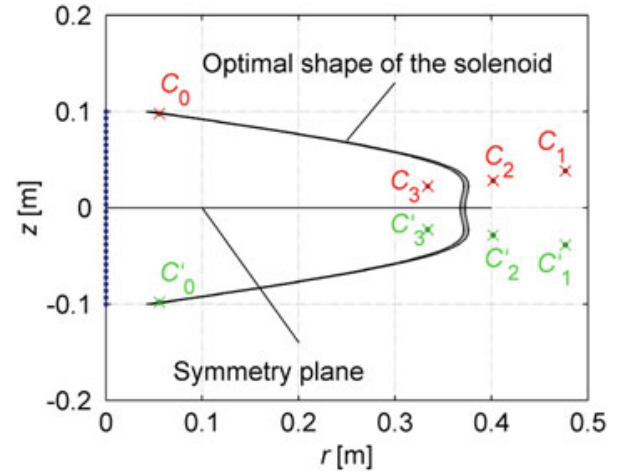

Fig. 10 Optimal shape of the solenoid for desired $H_{0}=1,500 \mathrm{~A} / \mathrm{m}$, $C_{i}$-positions of the Bezier curve control points

\subsection{Results for the genetic algorithm-based method}

In this method, the lower part of the solenoid's surface is built considering the symmetry plane of the solenoid and in figures, it is denoted by primed Bezier curve control points $C_{i}^{\prime}$. Similarly, like in previous method the desired magnetic fields values have been chosen as $H_{0}=1,500,2,000$ and $2,500 \mathrm{~A} / \mathrm{m}$, respectively. Also, there are two figures which present complete set of results obtained for each value of desired magnetic field $H_{0}$. For example, in Fig. 9 on the left, the cost function values $F$ after succeeding generations of GA for desired $H_{0}=1,500 \mathrm{~A} / \mathrm{m}$ have been presented (as described in Fig. 2). On the right, the relative error $\delta$ on solenoid's axis is shown for the minimal value of cost function $F$. The optimal shape of the solenoid for $H_{0}=1,500 \mathrm{~A} / \mathrm{m}$ has been shown in Fig. 10. Figures 11- 14 show the results of calculations for all the remaining desired values $H_{0}$.

It can be seen that in the best case (for desired $H_{0}=$ $2,000 \mathrm{~A} / \mathrm{m}$, Fig. 11, on the right) the maximal value of error $\delta$ does not exceed $1.6 \%$, and in the worst case (for desired $H_{0}=2,500 \mathrm{~A} / \mathrm{m}$, Fig. 13 , on the right) the maximal value of

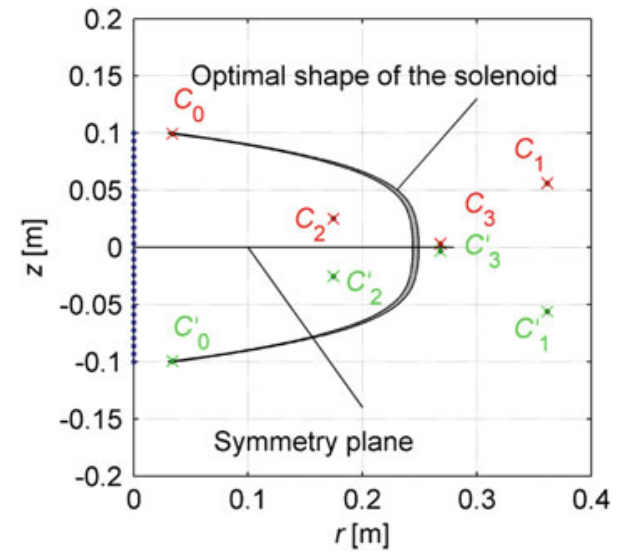

Fig. 12 Optimal shape of the solenoid for desired $H_{0}=2,000 \mathrm{~A} / \mathrm{m}$, $C_{i}$-positions of the Bezier curve control points

error $\delta$ is below $2.5 \%$. The obtained results are summarized in Table 4.

\section{Conclusions}

Two independent methods have been utilized in order to synthesize magnetic field of required distribution on solenoid's axis. The first iterative method is very sensitive to the initial values of design variables. In case of improperly set initial values, the iterations are not convergent and there is no solution to iterative expression (6). Additionally, the obtained shapes of the solenoid may be complex and difficult to build in practice (e.g. Fig. 8). The method based on GAs is better, because it gives simpler and smoother shapes in all considered cases. Although the time of calculations is slightly longer, there is no problem with convergence, and the solution's error is practically on the same level. The synthesis of a magnetic field in a finite
Fig. 11 On the left-cost function $F$ values after succeeding generations of GA for desired $H_{0}=2,000 \mathrm{~A} / \mathrm{m}$; on the right-relative error $\delta(\%)$
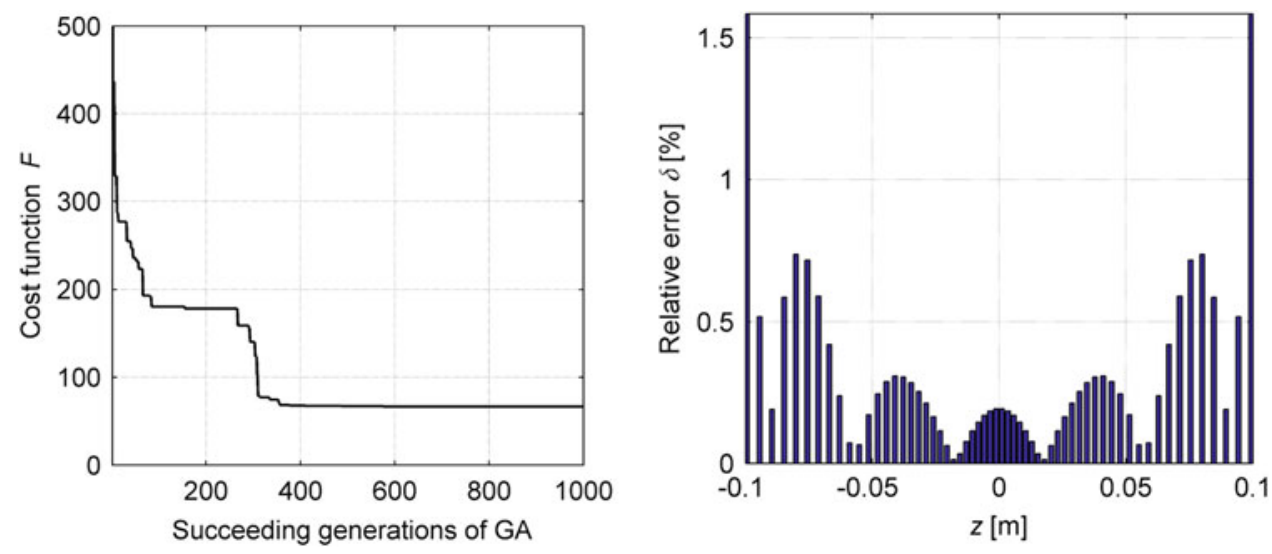
Fig. 13 On the left-cost function $F$ values after succeeding generations of $\mathrm{GA}$ for desired $H_{0}=2,500 \mathrm{~A} / \mathrm{m}$; on the right-relative error $\delta(\%)$
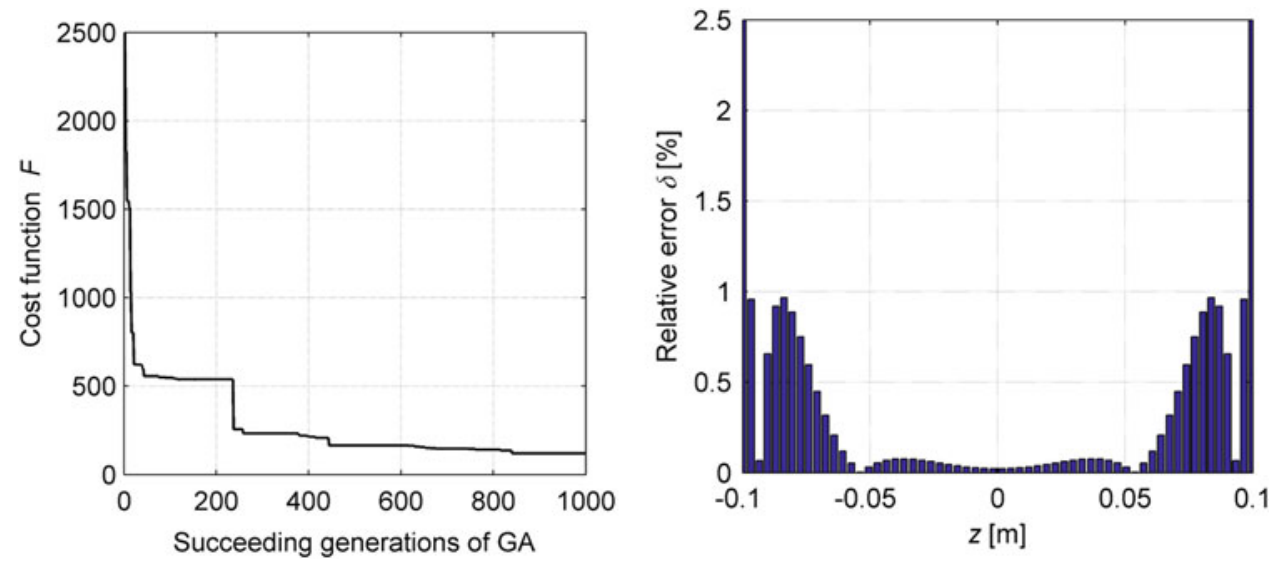

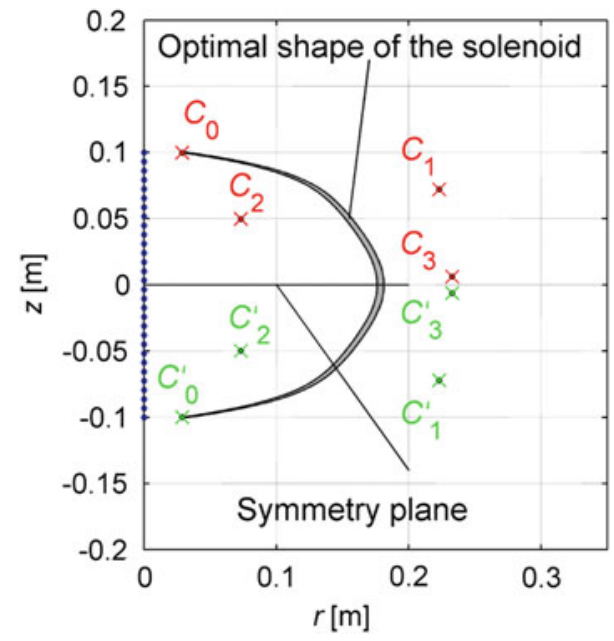

Fig. 14 Optimal shape of the solenoid for desired $H_{0}=2,500 \mathrm{~A} / \mathrm{m}$, $C_{i}$-positions of the Bezier curve control points

Table 4 Summarized results obtained by using Genetic Algorithm and Bezier curve based method

\begin{tabular}{lll}
\hline$H_{0}(\mathrm{~A} / \mathrm{m})$ & $\begin{array}{l}\delta_{\text {mean }} \\
(\%)\end{array}$ & $\begin{array}{l}\delta_{\max } \\
(\%)\end{array}$ \\
\hline 1,500 & 0.49 & 1.79 \\
2,000 & 0.30 & 1.58 \\
2,500 & 0.34 & 2.50 \\
\hline
\end{tabular}

three dimensional region will be the subject of the next paper.

Open Access This article is distributed under the terms of the Creative Commons Attribution Noncommercial License which permits any noncommercial use, distribution, and reproduction in any medium, provided the original author(s) and source are credited.

\section{References}

1. Ishiyama A, Hondoh M, Ishida N, Onuki T (1989) Optimal design of MRI magnets with magnetic shielding. IEEE Trans Magn 25(2):1885-1888
2. Kidane TK, Edelstein WA, Eagan TP, Taracila V, Baig TN, Cheng YuN, Brown RW (2006) Active-passive shielding for MRI acoustic noise reduction: network analysis. IEEE Trans Magn 42(12):3854-3860

3. Celozzi S, Araneo R, Lovat G (2008) Electromagnetic shielding. IEEE Press John Wiley, Italy

4. Ziolkowski M, Gratkowski S (2009) Active, passive and dynamic shielding of static and low frequency magnetic fields. In: XV International Symposium on Theoretical Electrical Engineering ISTET, June 22-24, Lubeck, Germany

5. Ziolkowski M, Gratkowski S (2011) A comparison of two formulations for magnetic field synthesis on a cylindrical solenoid's axis. Int J Appl Electromagn Mech (in press)

6. Sikora R, Purczynski J, Adamiak K (1978) The magnetic field synthesis on a cylinder solenoid axis by means of Tichonov's regularization method. Archiv fur Elektrotechnik 60:83-86

7. Tikhonov AN, Goncharsky AV, Stepanov VV, Yagola AG (1995) Numerical methods for the solution of ill-posed problems. Kluwer Academic Publishers, Dordrecht

8. Adamiak K (1980) Synthesis of homogeneous magnetic field in internal region of cylindrical solenoid. Archiv fur Elektrotechnik 62:75-79

9. Zhou P (1993) Numerical analysis of electromagnetic fields. Springer, Berlin

10. Adamiak K (1977) Tichonov's regularization method applied to the magnetic field on a solenoid axis synthesis. Archiv fur Elektrotechnik 59:19-22

11. Engl HW, Kugler P (2005) Multidisciplinary methods for analysis optimization and control complex systems. Springer, Berlin. Springer link Chapter: Nonlinear inverse problems: theoretical aspects and some industrial applications, see www.ipam.ucla.edu/ publications/invtut/invtut_hengl_pkugler.pdf

12. Doicu A, Schüssler O, Loyola D (2010) Constrained regularization methods for ozone profile retrieval from UV/VIS nadir spectrometers. J Quant Spectrosc Radiat Transf 111:907-916

13. Hansen PC (1992) Analysis of discrete ill-posed problems by means of the L-curve. SIAM Rev 34:561-580

14. Ziolkowski M, Gratkowski S, Palka R (2009) Solution of three dimensional inverse problem of magnetic induction tomography using Tikhonov regularization method. Int J Appl Electromagn Mech 30:245-253

15. Golub GH, Heath M, Wahba G (1978) Generalized cross-validation as a method for choosing a good ridge parameter. Technometrics 21:215-223

16. Park H, Shin S, Lee HA (1999) Tikhonov regularization scheme for system identification of continuous structures. In: Transactions of the 15th International Conference on Structural Mechanics in Reactor Technology (SMiRT-15), Seoul, Korea 
17. Vogel C (1996) Non-convergence of the L-curve regularization parameter selection method. Inverse Probl 12:535-547

18. Zeb A, Ingham DB, Lesnic D (2008) The method of fundamental solutions for a biharmonic inverse boundary determination problem. Comput Mech 42:371-379

19. Piegl L (1993) Fundamental developments of computer aided geometric design. Academic Press, San Diego
20. Ziolkowski M, Gratkowski S (2008) Genetic algorithm and Bezier curves based shape optimization of conducting shields for low frequency magnetic fields. IEEE Trans Magn 44:1086-1089

21. Haupt RL, Haupt SE (2004) Practical genetic algorithms, 2nd edn. Wiley, New York 